\title{
Severe adverse maternal outcomes among low risk women with planned home versus hospital births in the Netherlands: nationwide cohort study
}

\author{
OPEN ACCESS
}

\author{
Ank de Jonge midwife senior researcher ${ }^{1}$, Jeanette A J M Mesman physician assistant midwife ${ }^{2}$, \\ Judith Manniën senior researcher ${ }^{1}$, Joost J Zwart obstetrician ${ }^{3}$, Jeroen van Dillen obstetrician ${ }^{4}$, Jos \\ van Roosmalen professor ${ }^{25}$
}

${ }^{1}$ Department of Midwifery Science, AVAG and the EMGO Institute of Health and Care Research, VU University Medical Center, Amsterdam, Netherlands; ${ }^{2}$ Department of Obstetrics, Leiden University Medical Center, Leiden, Netherlands; ${ }^{3}$ Deventer Hospital, Deventer, Netherlands; ${ }^{4}$ Radboud University Nijmegen Medical Center, Nijmegen, Netherlands; ${ }^{5}$ Department of Medical Humanities, EMGO, VU University Medical Center, Netherlands

\begin{abstract}
Objectives To test the hypothesis that low risk women at the onset of labour with planned home birth have a higher rate of severe acute maternal morbidity than women with planned hospital birth, and to compare the rate of postpartum haemorrhage and manual removal of placenta.

Design Cohort study using a linked dataset.

Setting Information on all cases of severe acute maternal morbidity in the Netherlands collected by the national study into ethnic determinants of maternal morbidity in the netherlands (LEMMoN study), 1 August 2004 to 1 August 2006, merged with data from the Netherlands perinatal register of all births occurring during the same period.
\end{abstract}

Participants 146752 low risk women in primary care at the onset of labour.

Main outcome measures Severe acute maternal morbidity (admission to an intensive care unit, eclampsia, blood transfusion of four or more packed cells, and other serious events), postpartum haemorrhage, and manual removal of placenta.

Results Overall, 92333 (62.9\%) women had a planned home birth and $54419(37.1 \%)$ a planned hospital birth. The rate of severe acute maternal morbidity among planned primary care births was 2.0 per 1000 births. For nulliparous women the rate for planned home versus planned hospital birth was 2.3 versus 3.1 per 1000 births (adjusted odds ratio $0.77,95 \%$ confidence interval 0.56 to 1.06 ), relative risk reduction $25.7 \%$ (95\% confidence interval $-0.1 \%$ to $53.5 \%$ ), the rate of postpartum haemorrhage was 43.1 versus $43.3(0.92,0.85$ to 1.00 and $0.5 \%,-6.8 \%$ to $7.9 \%$ ), and the rate of manual removal of placenta was 29.0 versus $29.8(0.91,0.83$ to 1.00 and $2.8 \%,-6.1 \%$ to $11.8 \%)$. For parous women the rate of severe acute maternal morbidity for planned home versus planned hospital birth was 1.0 versus 2.3 per 1000 births $(0.43,0.29$ to
0.63 and $58.3 \%, 33.2 \%$ to $87.5 \%$ ), the rate of postpartum haemorrhage was 19.6 versus $37.6(0.50,0.46$ to 0.55 and $47.9 \%, 41.2 \%$ to $54.7 \%)$, and the rate of manual removal of placenta was 8.5 versus $19.6(0.41$, 0.36 to 0.47 and $56.9 \%, 47.9 \%$ to $66.3 \%$ ).

Conclusions Low risk women in primary care at the onset of labour with planned home birth had lower rates of severe acute maternal morbidity, postpartum haemorrhage, and manual removal of placenta than those with planned hospital birth. For parous women these differences were statistically significant. Absolute risks were small in both groups. There was no evidence that planned home birth among low risk women leads to an increased risk of severe adverse maternal outcomes in a maternity care system with well trained midwives and a good referral and transportation system.

\section{Introduction}

The relative safety of planned home births is a topic of continuous debate. ${ }^{1}$ Several studies have compared severe adverse perinatal outcomes among planned home births with those of planned hospital births. ${ }^{2-6}$ The rate of adverse perinatal outcomes was low and not significantly different in most studies, ${ }^{3-6}$ although slightly higher for primiparous women with planned home births in a recent large cohort study. ${ }^{2}$ The authors, however, disagreed about the interpretation of these results. ${ }^{267}$ Less evidence is available on the association between planned place of birth and maternal morbidity, especially severe adverse maternal outcomes, since these are rarer than severe adverse perinatal outcomes. Several studies have shown that at the onset of labour low risk women with planned home births have lower rates of referral to secondary care, augmentation, medical pain relief, operative delivery, postpartum haemorrhage, and episiotomy than women with planned hospital births. ${ }^{24589}$ 
Some have questioned the rationale of routine hospital birth for low risk women because of the exposure to overuse of medical interventions with potentially harmful effects. ${ }^{1}$ However, although the overall rate of maternal complications may be lower among planned home births, the delay due to transportation from home to hospital might lead to severe acute maternal morbidity. A previous Dutch study showed that the lower rate of medical interventions is not an important reason for women to choose a home birth, but sense of safety is a dominant reason to choose a hospital birth. ${ }^{10}$ Therefore, even though the rate of severe acute maternal morbidity is small, if the risk would be higher among planned home births this would probably be a reason for many women to choose a hospital birth. As far as we know, no studies have been large enough to study severe acute maternal morbidity among planned home births.

Of all Western countries, the Netherlands has the highest percentage of home births and is therefore ideally suited to study the association between planned place of birth and rare but severe outcomes. ${ }^{311}$ National obstetric, midwifery, and neonatal data are recorded in the Netherlands perinatal register. In addition, the national study into ethnic determinants of maternal morbidity in the netherlands (the LEMMoN study; Landelijke studie naar Etnische verschillen in Maternale Morbiditeit in Nederland) resulted in a database of all cases of severe acute maternal morbidity in the country over two years. ${ }^{12}$ Merging data from the national perinatal register and LEMMoN databases provided us with a unique opportunity to compare the rate of severe acute maternal morbidity among planned home births and planned hospital births. In addition, we compared the rate of postpartum haemorrhage and manual removal of placenta. The main hypothesis was that low risk women in primary care at the onset of labour with planned home birth have higher rates of severe acute maternal morbidity than those with planned hospital birth.

\section{Methods}

In the Netherlands, midwives in primary care provide care to low risk women. These are women with a singleton pregnancy of a fetus in cephalic presentation who do not have any medical or obstetric risk factors that are an indication for secondary care, such as previous caesarean section, and who start labour spontaneously between 37 and 42 weeks.

If complications or risk factors occur during pregnancy, labour, or after birth, women are referred to secondary care. After referral, women may receive care from clinical midwives, obstetricians, obstetric registrars, and obstetric nurses, under the final responsibility of an obstetrician. Obstetric interventions such as electronic fetal monitoring, augmentation, and medical pain relief only take place in secondary care. The indications for referral are laid out in the obstetric indication list. ${ }^{13}$ This list is revised regularly by a project group consisting of midwives, obstetricians, paediatricians, and general practitioners.

Women who are still in primary care at term can choose to give birth at home or in hospital, assisted by their primary care midwife. Women with a "medium risk" indication can give birth in primary care but are advised to give birth in hospital. The official medium risk indications according to the obstetric indication list are postpartum haemorrhage or retained placenta after a previous birth. ${ }^{13}$ Midwives may record other reasons for medium risk if they think it is better for a woman to give birth in hospital.

\section{Data linkage}

We combined the information from the datasets of the LEMMoN study and the national perinatal register. The methods of the LEMMoN study have been described in detail elsewhere. ${ }^{12}$ In short, all cases of severe acute maternal morbidity were collected from all 98 hospitals in the Netherlands over two years (1 August 2004 to 1 August 2006). Each month a local coordinator reported all cases, or the fact that there were no cases, via a web based form.

The national perinatal register database consists of data from three separate databases: one for primary care (national perinatal database-1), one for secondary care (national perinatal database-2), and one for paediatric care (national neonatal register). During 2004-06 an estimated 95-99\% of primary midwifery care practices and $99-100 \%$ of hospital based obstetric practices entered data into the perinatal register. ${ }^{14-16}$ The three datasets are combined into one national perinatal database via a validated linkage method. ${ }^{17} \mathrm{We}$ selected all data from the national perinatal register for the period in which the LEMMoN study took place.

In both databases we selected women with a singleton pregnancy without a history of caesarean section who gave birth between 37 and 42 weeks and had spontaneous onset of labour. We only included cases in the LEMMoN study if severe acute maternal morbidity occurred after the onset of labour.

Primary linkage of data from both datasets was based on date of birth of the baby plus or minus two days and date of birth of the woman. If there was more than one match or if date of birth of the baby was missing in one of the datasets, we used the following additional variables for matching: postpartum haemorrhage more than $1000 \mathrm{~mL}$, hospital number, and postal code. Two researchers (AJ and JMa) checked whether the data were well matched. We compared the characteristics of LEMMoN cases that were not linked with the national perinatal register with those that were linked.

We excluded women who were referred during labour from primary to secondary care but were missing the form from primary care, owing to important information, for example on their planned place of birth, being unavailable. We compared the characteristics of these women with the total sample to examine differences between the two groups.

\section{Study sample}

For the analyses we selected women who were in primary care at the onset of labour. We excluded women who were referred because of ruptured membranes for more than 24 hours without contractions since their planned place of birth did not have an effect on their labour process. To ensure that groups were as comparable as possible, we excluded all women with a record of a "medium risk" indication.

The study sample therefore consisted of women in primary care with a term singleton pregnancy without a medium risk indication, prolonged ruptured membranes without contractions, or any indication for secondary care at the onset of labour.

\section{Definition of variables}

The variable for planned place of birth comprised three categories: planned home birth, planned hospital birth, and unknown planned place of birth. At some point during pregnancy the midwives in primary care register women's planned place of birth in the national perinatal database- 1 . This information is missing for some women; midwives may forget to record the 
details or the women may not have made a decision on where to give birth until the onset of labour.

The main outcome variable was severe acute maternal morbidity, which was defined in the LEMMoN study in five different categories: admission to intensive care, uterine rupture, eclampsia or HELLP (haemolysis, elevated liver enzymes, and low platelet count) with liver haematoma, major obstetric haemorrhage (blood transfusion of four or more packed cells), and other severe acute maternal morbidity as diagnosed by the attending clinician. Secondary outcomes were the individual categories of severe acute maternal morbidity; we combined uterine rupture and other indications in the category "miscellaneous." Other secondary outcomes were postpartum haemorrhage more than $1000 \mathrm{~mL}$ and manual removal of placenta, both based on data from the perinatal register.

We identified the following confounders that may be associated with planned place of birth and with maternal complications: parity, gestational age, maternal age, ethnicity, and socioeconomic position. ${ }^{12} 1819$ Parity was coded as nulliparous or parous. Gestational age was divided into 37 to $37+6$ weeks, 38 to $40+6$ weeks, and $41+0$ to $41+6$ weeks. Maternal age was coded as less than 25 , between 25 and 34 , and 35 or older. The ethnicity classification is challenging in the perinatal register-for example, women of Turkish or Moroccan background are both classified as "Mediterranean" and women of African origin are classified by some midwives as "creole" and by others as "other." We therefore categorised ethnicity as Dutch and non-Dutch. Socioeconomic position was derived from social status scores based on postal codes developed by the National Institute for Social Research based on income, employment, and level of education. These scores were divided into low (below 25th centile), medium (between 25th and 75th centile), and high (above 75th centile).

Augmentation of labour with oxytocin and operative delivery (caesarean section, vacuum, or forceps delivery) have been associated with adverse maternal outcomes. ${ }^{12}{ }^{18}{ }^{20}$ In a secondary analysis we therefore controlled the results for augmentation of labour and operative delivery (vacuum, forceps, or caesarean section).

\section{Data analyses}

We used SAS version 9.2 to merge data, and analysed the data using SPSS version 19.0. Within each planned place of birth category we calculated the number and percentage of the primary and secondary outcomes. We performed logistic regression analyses only for severe acute maternal morbidity, blood transfusion of four or more packed cells, postpartum haemorrhage, and manual removal of placenta, because of a low number of events in the other outcomes; these analyses were done for nulliparous and parous women separately and for planned home births versus planned hospital births. For all of these outcomes we present the crude odds ratios and 95\% confidence intervals. We used multivariable logistic regression analyses to control for potential confounders, resulting in adjusted odds ratios with $95 \%$ confidence intervals. We also present relative risk reductions with $95 \%$ confidence intervals. Subsequently, the associations between planned place of birth and severe acute maternal morbidity were controlled for augmentation of labour with oxytocin and operative delivery (both as binary variables). We excluded missing data because they were less than $5 \%$ for all variables.

For the main analyses we used the perinatal register definition of onset of labour in primary or secondary care. Onset of labour is defined in the register based on information from the databases for primary and secondary care, but this information is not always consistent. We conducted sensitivity analyses for women without discrepancies between data from primary and secondary care for this variable and for onset of labour based on the national perinatal database- 1 only.

\section{Results \\ Linkage of data}

During the study period, 240400 women who had no previous caesarean section, a singleton pregnancy, and a spontaneous onset of labour between 37 and 42 weeks' gestation were recorded in the national perinatal register. In the LEMMoN study, 706 women met these criteria and had severe acute maternal morbidity after the onset of labour (27.7\% of all women with severe acute maternal morbidity) (figure $\downarrow$ ). Of these, 56 could not be linked to data in the perinatal register (7.9\%). Women with severe acute maternal morbidity who were linked to the perinatal register did not differ significantly for type of severe acute maternal morbidity, parity, and ethnicity from those that were not linked to the register.

Of the total linked data, 10101 (4.2\%) women were referred during or after labour but were missing the national perinatal database- 1 form and 52 of the women in this category had severe acute maternal morbidity. Compared with all women who were referred during or after labour these women were more likely to be parous $(31.2 \% v 30.0 \%)$ and of Dutch ethnicity $(83.4 \% v$ $78.7 \%$ ). There were no significant differences between these groups in incidence and type of severe acute maternal morbidity. The linked dataset contained information on 230299 women, of whom 598 (2.6 per 1000) had severe acute maternal morbidity. Of these, 172973 started labour in primary care (severe acute maternal morbidity, $\mathrm{n}=364$ ), and for 439 women (severe acute maternal morbidity, $n=1$ ) the level of care at the start of labour was unknown.

\section{Study population}

Of the women in primary care at the onset of labour, planned place of birth was unknown for 18070 and these women were not included in the analyses (fig 1). Another 2112 women were excluded because they had a "medium risk" indication. Of these, $1248(59.1 \%)$ had a history of retained placenta or postpartum haemorrhage and the others had various indications such as "no prenatal care" and "use of medication (not further specified)." An additional 6039 women were not included because they were referred for prolonged ruptured membranes without contractions. Of the remaining 146752 women in primary care at the onset of labour, $92333(62.9 \%)$ had a planned home birth and 54419 $(37.1 \%)$ had a planned hospital birth (table $1 \Downarrow$ ). Women with planned home birth compared with those with planned hospital birth were more likely to be parous, less likely to give birth between $37+0$ and $37+6$ weeks' gestation, and more likely to give birth between $41+0$ and $41+6$ weeks; they were less often younger than 25 years, more often aged between 25 and 34 years, more often of Dutch origin, and less often of a lower socioeconomic position.

\section{Adverse maternal outcomes}

Of all women included in the analyses, 288 (2.0 per 1000) had severe acute maternal morbidity (table $2 \Downarrow$ ). Among planned home births, severe acute maternal morbidity occurred in 141 women (1.5 per 1000) and among planned hospital births in 147 women (2.7 per 1000). Most of the affected women had a blood transfusion of four or more packed cells. Other causes 
were rare. Postpartum haemorrhage was the most common adverse maternal outcome and this occurred among 2699 (29.2 per 1000) planned home births and among 2172 (39.9 per 1000) planned hospital births.

Adverse outcomes were less common among planned home births than among planned hospital births, but differences were only statistically significant for parous women (table $3 \Downarrow$ ).

Among nulliparous women outcomes for planned home versus planned hospital births were: severe acute maternal morbidity adjusted odds ratio 0.77 (95\% confidence interval 0.56 to 1.06 ) and relative risk reduction $25.7 \%$ (95\% confidence interval $-0.1 \%$ to $53.5 \%)$, blood transfusion of four or more packed cells 0.90 (0.65 to 1.27$)$ and $14.5 \%$ ( $-14.7 \%$ to $45.8 \%)$, postpartum haemorrhage 0.92 ( 0.85 to 1.00$)$ and $0.5 \%$ ( $-6.8 \%$ to $7.9 \%$ ), and manual removal of placenta 0.91 (0.83 to 1.00$)$ and $2.8 \%$ ( $-6.1 \%$ to $11.8 \%)$. Among parous women outcomes for planned home versus hospital births were: severe acute maternal morbidity adjusted odds ratio 0.43 (95\% confidence interval 0.29 to 0.63 ), blood transfusion of four or more packed cells 0.45 (0.30 to 0.68$)$, postpartum haemorrhage 0.50 (0.46 to 0.55 ), and manual removal of placenta 0.41 (0.36 to 0.47 ).

\section{Sensitivity analyses and adjustment for medical interventions}

Sensitivity analyses showed similar results for all outcomes in table 3 (data not shown). In some of the sensitivity analyses, differences just reached statistical significance that did not in the main analyses. For example, for the comparison of severe acute maternal morbidity, if only women without discrepancies in onset of labour between the data forms from primary and secondary care were selected the adjusted odds ratio for planned home versus planned hospital birth in nulliparous women was 0.63 (95\% confidence interval 0.44 to 0.88 ) and in parous women was 0.46 (0.30 to 0.69 ). If onset of labour was based on the national perinatal database- 1 form only, the differences in severe acute maternal morbidity, postpartum haemorrhage, and manual removal of placenta became significant for nulliparous women: 0.72 ( 0.53 to 0.99$), 0.90$ (0.83 to 0.97$)$, and 0.88 (0.80 to 0.96$)$, respectively.

Fewer women with planned home births compared with planned hospital births received augmentation of labour (nulliparous women $22.9 \% v 27.5 \%$ and parous women $3.4 \% v 7.8 \%$, respectively) and had an operative delivery (nulliparous women $23.1 \% v 24.7 \%$ and parous women $1.6 \%$ v $3.2 \%$ ). The comparison of severe acute maternal morbidity controlled for augmentation of labour and operative delivery for planned home versus planned hospital births among nulliparous women gave an adjusted odds ratio of 0.80 (0.58 to 1.10), which is an increase of $3.9 \%$ in odds ratio. For parous women the adjusted odds ratio for severe acute maternal morbidity after controlling for these interventions was 0.47 (0.32 to 0.69$)$, which is an increase of $9.3 \%$ in odds ratio.

\section{Discussion}

Low risk women in primary care at the onset of labour who planned to give birth at home had lower rates of severe acute maternal morbidity, postpartum haemorrhage, and manual removal of placenta compared with women who planned to give birth in hospital, but the differences were only statistically significant for parous women. Odds ratios for severe acute maternal morbidity changed slightly when we adjusted the results for medical interventions, and more so for parous than for nulliparous women.

\section{Strengths and limitations of this study}

A major strength of our study is the large sample size and the fact that all cases of severe acute maternal morbidity that occurred in all hospitals in the Netherlands were collected meticulously over two years. As far as we are aware, this is the largest study to date into the association between planned place of birth and severe adverse maternal outcomes.

Our study has some limitations as well. Firstly, because we used registration data, some were missing or may have been misclassified. For example, information on the variable "start of labour in primary or secondary care" was not always consistent between midwifery and obstetric registration. However, sensitivity analyses using different definitions of this variable generated similar results. In addition, 10101 women were excluded because their national perinatal database- 1 form was missing when they were referred during labour. Some of these women were cared for by general practitioners or midwives who do not participate in the national perinatal registration. In particular, general practitioners who still practise midwifery are often located in rural areas. This may explain the higher rate of parous women and women of Dutch ethnicity among those with a missing national perinatal database- 1 form. For 18070 women planned place of birth at the onset of labour was unknown. Their rate of severe acute maternal morbidity was comparable to that of women who planned hospital births. Even if all of these women would have a planned home birth or, alternatively, if all of them would have a planned hospital birth, the strength of the associations would have changed but the results would have been in the same direction.

Secondly, we collected the data from 2004 to 2006 and theoretically midwifery management and women's characteristics may have changed. However, we have no reason to believe that at present planned home birth leads to more unfavourable maternal outcomes. For example, the percentage of women with a singleton pregnancy who were older than 35 years only increased from $20.5 \%$ in 2004 to $21.7 \%$ in 2006 and this percentage was $21.4 \%$ in $2010 .{ }^{14-1621}$ Besides, we controlled the results for differences in maternal age.

Thirdly, although none of the women who started labour in primary care should have had an indication for secondary care according to the obstetric indication list, there may still have been differences in risk profiles between women who planned labour at home versus in hospital. We corrected the analyses for known risk factors, such as maternal age and ethnicity. Adjusting the results regarding severe acute maternal morbidity for augmentation of labour and operative delivery only led to a small reduction in the differences. This means that medical interventions explain some of the differences in severe acute maternal morbidity, which is consistent with earlier studies that showed higher rates of adverse maternal outcomes among women with medical interventions. ${ }^{12}{ }^{18}{ }^{20}$ However, the fact that odds ratios for adverse maternal outcomes were much lower for parous women than for nulliparous women, suggests that other factors played an important part. Those women who had a relatively difficult previous birth may have been more likely to plan a hospital birth next time, even if there was no official medical indication. If so, this self selection may have resulted in better outcomes among women with planned home birth. In addition, there may have been residual confounding owing to differences in characteristics that could not be identified. For example, we had no information on body mass index. Although a high body mass index is not an official medium risk indication according to the obstetric indication list, midwives may have advised these women to give birth in hospital. They may have ticked the medium risk box but they could not record body mass 
index as the reason for medium risk in the national perinatal database- $1 .^{13}$

Nevertheless, our hypothesis that low risk women at the onset of labour who planned birth at home would have a higher rate of severe acute maternal morbidity compared with women who planned birth in hospital was not confirmed. Women with planned home birth had lower rates of all adverse maternal outcomes, albeit not significantly so for nulliparous women. This is consistent with other studies that found lower rates of maternal morbidity among planned home births. ${ }^{24522}$ Concern about safety is an important reason for women to choose hospital birth, and even more so for their partners. ${ }^{1023}$ They worry especially about transportation to hospital in case of an emergency. However, although the referral rate during labour is high in the Netherlands, only $3.4 \%$ of women are referred for urgent reasons. ${ }^{24}$ Our results suggest that planned home birth for low risk women is not associated with an increased risk of adverse maternal outcomes despite the possible delay in case of an emergency. Previous studies have not shown higher risks of severe adverse perinatal outcomes either for planned home births compared with planned hospital births in the Netherlands. ${ }^{36}$ We should emphasise that our results may only apply to regions where midwives are well trained to assist women at home births and where facilities for transfer of care and transportation in case of emergencies are adequate. In 2009, $82 \%$ of women were in hospital within 45 minutes from the moment a midwife called an ambulance in an emergency situation..$^{25}$ The average time was 35 minutes (standard deviation 12 minutes). Travelling time to hospital is important for the safety of all births, regardless of planned place of birth. A Dutch study showed that the incidence of adverse perinatal outcomes was higher if travel time from home to hospital was more than 20 minutes, but differences were only statistically significant for women in secondary care at the onset of labour. ${ }^{26}$

Planned hospital births are also associated with risks. The rate of medical interventions is lower for planned home versus planned hospital births among low risk women; for example, odds ratios for caesarean section varied between 0.31 and 0.76 in different studies. ${ }^{2459} \mathrm{It}$ is important to limit the use of caesarean section because of its association with various adverse outcomes at the current birth, and the risk of uterine scar rupture during the next pregnancy and birth. ${ }^{12} 18{ }^{20-29}$ However, again selection bias may play a part despite all women in these studies being considered at "low risk." Although more women with planned hospital birth may have needed interventions to ensure a good perinatal outcome, considering the large size of the differences in the rate of medical interventions between the groups, it is unlikely that these can be explained by a difference in risk profile only.

The fact that we did not find higher rates of severe acute maternal morbidity among planned home births should not lead to complacency. Every avoidable adverse maternal outcome is one too many. An audit of maternal morbidity should be used to learn from every case of severe acute maternal morbidity to improve care, optimise the risk selection system, and prevent future severe acute maternal morbidity from happening. ${ }^{30}$

\section{Conclusion}

Our study showed a lower risk of severe acute maternal morbidity, postpartum haemorrhage, and manual removal of placenta among low risk women in primary care at the onset of labour with planned home versus planned hospital births. These differences were statistically significant for parous women. We found no evidence that planned home birth among low risk women leads to an increased risk of severe adverse maternal outcomes in a maternity care system with well trained midwives and a good referral and transportation system.

We thank the Netherlands perinatal registry for the use of the national database.

Contributors: AJ conceived the study, wrote the article, and is guarantor of the study. JMe and AJ conducted the analyses. JMa linked the datasets. All authors contributed to interpretation of the data, critically revised earlier drafts of the paper for important intellectual content, and gave final approval of the version to be published. The researchers had access to all the research data.

Funding: This study was funded with a career grant (VENI) from ZonMw. The funder had no role in any aspect of the study.

Competing interests: All authors have completed the ICMJE uniform disclosure form at www.icmje.org/coi_disclosure.pdf (available on request from the corresponding author) and declare: no support from any organisation for the submitted work; no financial relationships with any organisations that might have an interest in the submitted work in the previous three years; no other relationships or activities that could appear to have influenced the submitted work.

Ethical approval: The ethical committee of VU University Medical Center confirmed that ethical approval was not necessary for this study (reference No 11/399).

Data sharing: No additional data available.

Olsen O, Clausen JA. Planned hospital birth versus planned home birth. Cochrane Database Syst Rev 2012;(9):CD000352.

2 Birthplace in England Collaborative Group. Perinatal and maternal outcomes by planned place of birth for healthy women with low risk pregnancies: the Birthplace in England national prospective cohort study. BMJ 2011;343:d7400.

3 De Jonge A, Van der Goes BY, Ravelli AC, Amelink-Verburg MP, Mol BW, Nijhuis JG, et al. Perinatal mortality and morbidity in a nationwide cohort of 529,688 low-risk planned home and hospital births. BJOG 2009;116:1177-84.

4 Hutton EK, Reitsma AH, Kaufman K. Outcomes associated with planned home and planned hospital births in low-risk women attended by midwives in Ontario, Canada, 2003-2006: a retrospective cohort study. Birth 2009;36:180-9.

5 Janssen PA, Saxell L, Page LA, Klein MC, Liston RM, Lee SK. Outcomes of planned home birth with registered midwife versus planned hospital birth with midwife or physician. CMAJ 2009;181:377-83

6 Van der Kooy J, Poeran J, De Graaf JP, Birnie E, Denktass S, Steegers EA, et al. Planned home compared with planned hospital births in the Netherlands: intrapartum and early neonatal death in low-risk pregnancies. Obstet Gynecol 2011;118:1037-46.

7 Offerhaus P, Rijnders M, De Jonge A, De Miranda E. Planned home compared with planned hospital births in the Netherlands: intrapartum and early neonatal death in low-risk pregnancies. Obstet Gynecol 2012;119(2 Pt 1):387-8.

8 Davis D, Baddock S, Pairman S, Hunter M, Benn C, Wilson D, et al. Planned place of birth in New Zealand: does it affect mode of birth and intervention rates among low-risk women? Birth 2011;38:111-9.

9 Lindgren HE, Radestad IJ, Christensson K, Hildingsson IM. Outcome of planned home births compared to hospital births in Sweden between 1992 and 2004. A population-based register study. Acta Obstet Gynecol Scand 2008;87:751-9.

10 Van Haaren-Ten Haken T, Hendrix M, Nieuwenhuijze M, Bude L, De Vries R, Nijhuis J. Preferred place of birth: characteristics and motives of low-risk nulliparous women in the Netherlands. Midwifery 2012;28:609-18.

11 Christiaens W, Nieuwenhuijze MJ, De Vries R. Trends in the medicalisation of childbirth in Flanders and the Netherlands. Midwifery 2013:29:e1-8.

12 Zwart JJ, Richters JM, Ory F, De Vries JI, Bloemenkamp KW, van Roosmalen J. Severe maternal morbidity during pregnancy, delivery and puerperium in the Netherlands: a nationwide population-based study of 371,000 pregnancies. BJOG 2008;115:842-50.

13 Obstetric Vademecum. Final report of the Maternity Care Committee of the College of health insurance companies. [Verloskundig Vademecum. Eindrapport van de Commissie Verloskunde van het College voor zorgverzekeringen]. De Koninklijke Nederlandse Organisatie van Verloskundigen (KNOV), De Landelijke Huisartsen Vereniging (LHV), De Nederlandse Vereniging voor Obstetrie en Gynaecologie (NVOG), Zorgverzekeraars Nederland (ZN), Inspectie voor de Gezondheidszorg, eds. 2003. Diemen.

14 Stichting Perinatale Registratie Nederland. Perinatal Care in the Netherlands 2004 [Perinatale zorg in Nederland 2004]. 2007. Stichting Perinatale Registratie Nederland.

15 Stichting Perinatale Registratie Nederland. Perinatal Care in the Netherlands 2005 [Perinatale Zorg in Nederland 2005]. 2008. Stichting Perinatale Registratie Nederland.

16 Stichting Perinatale Registratie Nederland. Perinatal Care in the Netherlands 2006 [Perinatale zorg in Nederland 2006]. 2008. Stichting Perinatale Registratie Nederland.

17 Meray N, Reitsma JB, Ravelli AC, Bonsel GJ. Probabilistic record linkage is a valid and transparent tool to combine databases without a patient identification number. J Clin Epidemiol 2007:60:883-91.

18 Kramer MS, Dahhou M, Vallerand D, Liston R, Joseph KS. Risk factors for postpartum hemorrhage: can we explain the recent temporal increase? J Obstet Gynaecol Can 2011;33:810-9.

19 Zwart JJ, Jonkers MD, Richters A, Ory F, Bloemenkamp KW, Duvekot JJ, et al. Ethnic disparity in severe acute maternal morbidity: a nationwide cohort study in the Netherlands. Eur J Public Health 2011;21:229-34. 


\section{What is already known on this topic}

Low risk women with planned home birth at the onset of labour have lower rates of referral from primary to secondary care during labour, augmentation, medical pain relief, operative delivery, postpartum haemorrhage, and episiotomy than those with planned hospital birth Studies so far have been too small to compare severe acute maternal morbidity between planned home birth and planned hospital birth among low risk women

\section{What this study adds}

Low risk women in primary care with planned home birth at the onset of labour had a lower rate of severe acute maternal morbidity, postpartum haemorrhage, and manual removal of placenta than those with planned hospital birth

These differences were statistically significant for parous women

There was no evidence that planned home birth among low risk women leads to an increased risk of severe adverse maternal outcomes in a maternity care system with well trained midwives and a good referral and transportation system

20 Van Dillen J, Zwart JJ, Schutte J, Bloemenkamp KW, Van Roosmalen J. Severe acute maternal morbidity and mode of delivery in the Netherlands. Acta Obstet Gynecol Scand 2010;89:1460-5.

21 Stichting Perinatale Registratie Nederland. Perinatal Care in the Netherlands 2010 [Perinatale Zorg in Nederland 2010]. 2013. Stichting Perinatale Registrat

22 Blix E, Huitfeldt AS, Oian P, Straume B, Kumle M. Outcomes of planned home births and planned hospital births in low-risk women in Norway between 1990 and 2007: a retrospective cohort study. Sex Reprod Healthc 2012;3:147-53.

23 Bedwell C, Houghton G, Richens Y, Lavender T. 'She can choose, as long as I'm happy with it': a qualitative study of expectant fathers' views of birth place. Sex Reprod Healthc 2011;2:71-75.

24 Amelink-Verburg MP, Verloove-Vanhorick SP, Hakkenberg RM, Veldhuijzen IM, Bennebroek GJ, Buitendijk SE. Evaluation of 280,000 cases in Dutch midwifery practices: a descriptive study. BJOG 2008;115:570-8.

25 Kommer GJ, Zwakhals SLN. Time in ambulance care. Analysis of emergency journeys in 2009. [Tijdsduren in ambulancezorg. Analyse van spoedinzetten in 2009.]. RIVM, ed. RIVM Briefrapport 270482001/2010. 2010.

26 Ravelli AC, Jager KJ, De Groot MH, Erwich JJ, Rijninks-van Driel GC, Tromp M, et al. Travel time from home to hospital and adverse perinatal outcomes in women at term in the Netherlands. BJOG 2011;118:457-65.
27 Declercq E, Barger M, Cabral HJ, Evans SR, Kotelchuck M, Simon C, et al. Maternal outcomes associated with planned primary cesarean births compared with planned vaginal births. Obstet Gynecol 2007;109:669-77.

28 Declercq E, Cunningham DK, Johnson C, Sakala C. Mothers' reports of postpartum pain associated with vaginal and cesarean deliveries: results of a national survey. Birth 2008;35:16-24.

29 Fitzpatrick KE, Kurinczuk JJ, Alfirevic Z, Spark P, Brocklehurst P, Knight M. Uterine rupture by intended mode of delivery in the UK: a national case-control study. PLOS Med 2012;9:e1001184.

30 Van Dillen J, Mesman JAJM, Zwart JJ, Bloemenkamp KW, Van Roosmalen J. Introducing maternal morbidity audit in the Netherlands. BJOG 2010;117:416-21.

\section{Cite this as: BMJ 2013;346:f3263}

This is an Open Access article distributed in accordance with the Creative Commons Attribution Non Commercial (CC BY-NC 3.0) license, which permits others to distribute, remix, adapt, build upon this work non-commercially, and license their derivative works on different terms, provided the original work is properly cited and the use is non-commercial. See: http://creativecommons.org/licenses/by-nc/3.0/. 


\section{Tables}

Table 1 | Characteristics of low risk women in primary care at onset of labour

\begin{tabular}{|c|c|c|c|}
\hline \multirow[t]{2}{*}{ Characteristics } & \multirow[t]{2}{*}{ Total $(n=146752)$} & \multicolumn{2}{|c|}{ Planned place of birth at onset of labour } \\
\hline & & Home $(n=92333)$ & Hospital $(n=54419)$ \\
\hline \multicolumn{4}{|l|}{ Parity: } \\
\hline 0 & $65227(44.4)$ & $38728(41.9)$ & $26499(48.7)$ \\
\hline $1+$ & $81521(55.6)$ & $53602(58.1)$ & $27919(51.3)$ \\
\hline Missing data & $4(0)$ & - & - \\
\hline \multicolumn{4}{|l|}{ Gestational age: } \\
\hline $37+0$ to $37+6$ & $5700(3.9)$ & $3404(3.7)$ & $2296(4.2)$ \\
\hline $38+0$ to $40+6$ & $107763(73.4)$ & $67507(73.1)$ & $40256(74.0)$ \\
\hline $41+0$ to $41+6$ & $33289(22.7)$ & $21422(23.2)$ & $11867(21.8)$ \\
\hline \multicolumn{4}{|c|}{ Maternal age (years): } \\
\hline$<25$ & $18549(12.6)$ & $9142(9.9)$ & $9407(17.3)$ \\
\hline $25-34$ & $101691(69.3)$ & $66554(72.1)$ & $35137(64.6)$ \\
\hline$\geq 35$ & $26498(18.1)$ & $16630(18.0)$ & $9868(18.1)$ \\
\hline Missing data & $14(0)$ & - & - \\
\hline \multicolumn{4}{|l|}{ Ethnicity: } \\
\hline Dutch & $119755(82.0)$ & $83629(90.9)$ & 36126 (66.9) \\
\hline Non-Dutch & $26289(18.1)$ & $8385(9.1)$ & $17904(33.1)$ \\
\hline Missing data & $708(0.5)$ & - & - \\
\hline \multicolumn{4}{|c|}{ Socioeconomic position: } \\
\hline High & 35567 (24.6) & $23243(25.5)$ & $12324(23.0)$ \\
\hline Medium & 66419 (45.9) & $45320(49.7)$ & $21099(39.4)$ \\
\hline Low & $42861(29.6)$ & $22671(24.8)$ & $20190(37.7)$ \\
\hline Missing data & $1905(1.3)$ & - & - \\
\hline
\end{tabular}

For all characteristics $\mathrm{P}<0.001$. 
Table 2/ Severe acute maternal morbidity, postpartum haemorrhage, and manual removal of placenta in low risk births starting in primary care: total group

\begin{tabular}{lccc} 
Outcomes & \multicolumn{3}{c}{ No with outcome (No/1000 women) } \\
\cline { 2 - 4 } Severe acute maternal morbidity & Total $(\mathbf{n = 1 4 6 ~ 7 5 2 )}$ & Planned home birth ( $\mathbf{n = 9 2 ~ 3 3 3 ) ~ P l a n n e d ~ h o s p i t a l ~ b i r t h ~ ( ~} \mathbf{n = 5 4 ~ 4 1 9 )}$ \\
\hline Admission to intensive care unit & $288(2.0)$ & $141(1.5)$ & $147(2.7)$ \\
\hline Eclampsia or severe HELLP syndrome & $19(0.1)$ & $32(0.3)$ & $38(0.7)$ \\
\hline Blood transfusion $\geq 4$ packed cells & $256(1.7)$ & $8(0.1)$ & $11(0.2)$ \\
\hline Postpartum haemorrhage $(>1000 \mathrm{~mL})$ & $4871(33.2)$ & $2699(29.2)$ & $2172(39.9)$ \\
\hline Manual removal of placenta & $2865(19.5)$ & $1550(16.8)$ & $1315(24.2)$
\end{tabular}

HELLP=haemolysis, elevated liver enzymes, and low platelet count.

Missing data: postpartum haemorrhage 1234 (0.8\%), manual removal of placenta $2106(1.4 \%)$.

Women could have more than one type of severe acute maternal morbidity. 
Table 3/ Severe acute maternal morbidity, postpartum haemorrhage, and manual removal of placenta among low risk nulliparous and parous women starting labour in primary care

\begin{tabular}{|c|c|c|c|c|}
\hline \multirow[t]{2}{*}{ Variables } & \multicolumn{2}{|c|}{ Nulliparous women ( $n=65227$ ) } & \multicolumn{2}{|c|}{ Parous women $(n=81521)$} \\
\hline & Planned home birth ( $n=38728$ & $\begin{array}{l}\text { Planned hospital birth } \\
\qquad(\mathrm{n}=26499)\end{array}$ & Planned home birth $(n=53602)$ & $\begin{array}{l}\text { Planned hospital birth } \\
\qquad(\mathrm{n}=27 \text { 919) }\end{array}$ \\
\hline \multicolumn{5}{|l|}{ Severe acute maternal morbidity: } \\
\hline No (No/1000) & $89(2.3)$ & $82(3.1)$ & $52(1.0)$ & $65(2.3)$ \\
\hline Crude odds ratio $(95 \% \mathrm{Cl})$ & $0.74(0.55$ to 1.00$)$ & Reference & $0.42(0.29$ to 0.60$)$ & Reference \\
\hline Adjusted odds ratio $(95 \% \mathrm{Cl})$ & 0.77 (0.56 to 1.06$)$ & Reference & 0.43 (0.29 to 0.63$)$ & Reference \\
\hline Relative risk reduction $(\%, 95 \% \mathrm{Cl})$ & $25.7(-0.1$ to 53.5$)$ & Reference & 58.3 (33.2 to 87.5$)$ & Reference \\
\hline \multicolumn{5}{|l|}{ Blood transfusion $\geq 4$ packed cells: } \\
\hline No $(\mathrm{No} / 1000)$ & $85(2.2)$ & $68(2.6)$ & $49(0.9)$ & $54(1.9)$ \\
\hline Crude odds ratio $(95 \% \mathrm{Cl})$ & $0.86(0.62$ to 1.18$)$ & Reference & $0.47(0.32$ to 0.70$)$ & Reference \\
\hline Adjusted odds ratio $(95 \% \mathrm{Cl})$ & $0.90(0.65$ to 1.27$)$ & Reference & $0.45(0.30$ to 0.68$)$ & Reference \\
\hline Relative risk reduction $(\%, 95 \% \mathrm{Cl})$ & $14.5(-14.7$ to 45.8$)$ & Reference & 52.7 (24.9 to 85.3$)$ & Reference \\
\hline \multicolumn{5}{|l|}{ Postpartum haemorrhage: } \\
\hline No (No/1000) & $1655(43.1)$ & $1134(43.3)$ & $1044(19.6)$ & $1038(37.6)$ \\
\hline Crude odds ratio $(95 \% \mathrm{Cl})$ & $1.0(0.92$ to 1.07$)$ & Reference & $0.51(0.47$ to 0.56$)$ & Reference \\
\hline Adjusted odds ratio $(95 \% \mathrm{Cl})$ & $0.92(0.85$ to 1.00$)$ & Reference & 0.50 (0.46 to 0.55$)$ & Reference \\
\hline Relative risk reduction $(\%, 95 \% \mathrm{Cl})$ & $0.5(-6.8$ to 7.9$)$ & Reference & $47.9(41.2$ to 54.7$)$ & Reference \\
\hline \multicolumn{5}{|l|}{ Manual removal of placenta: } \\
\hline No (No/1000) & $1099(29.0)$ & $773(29.8)$ & $451(8.5)$ & $542(19.6)$ \\
\hline Crude odds ratio $(95 \% \mathrm{Cl})$ & $0.97(0.89$ to 1.07$)$ & Reference & $0.43(0.38$ to 0.48$)$ & Reference \\
\hline Adjusted odds ratio $(95 \% \mathrm{Cl})$ & $0.91(0.83$ to 1.00$)$ & Reference & $0.41(0.36$ to 0.47$)$ & Reference \\
\hline Relative risk reduction $(\%, 95 \% \mathrm{Cl})$ & $2.8(-6.1$ to 11.8$)$ & Reference & 56.9 (47.9 to 66.3$)$ & Reference \\
\hline
\end{tabular}

Adjusted relative risks adjusted for variables in table 1. 


\section{Figure}

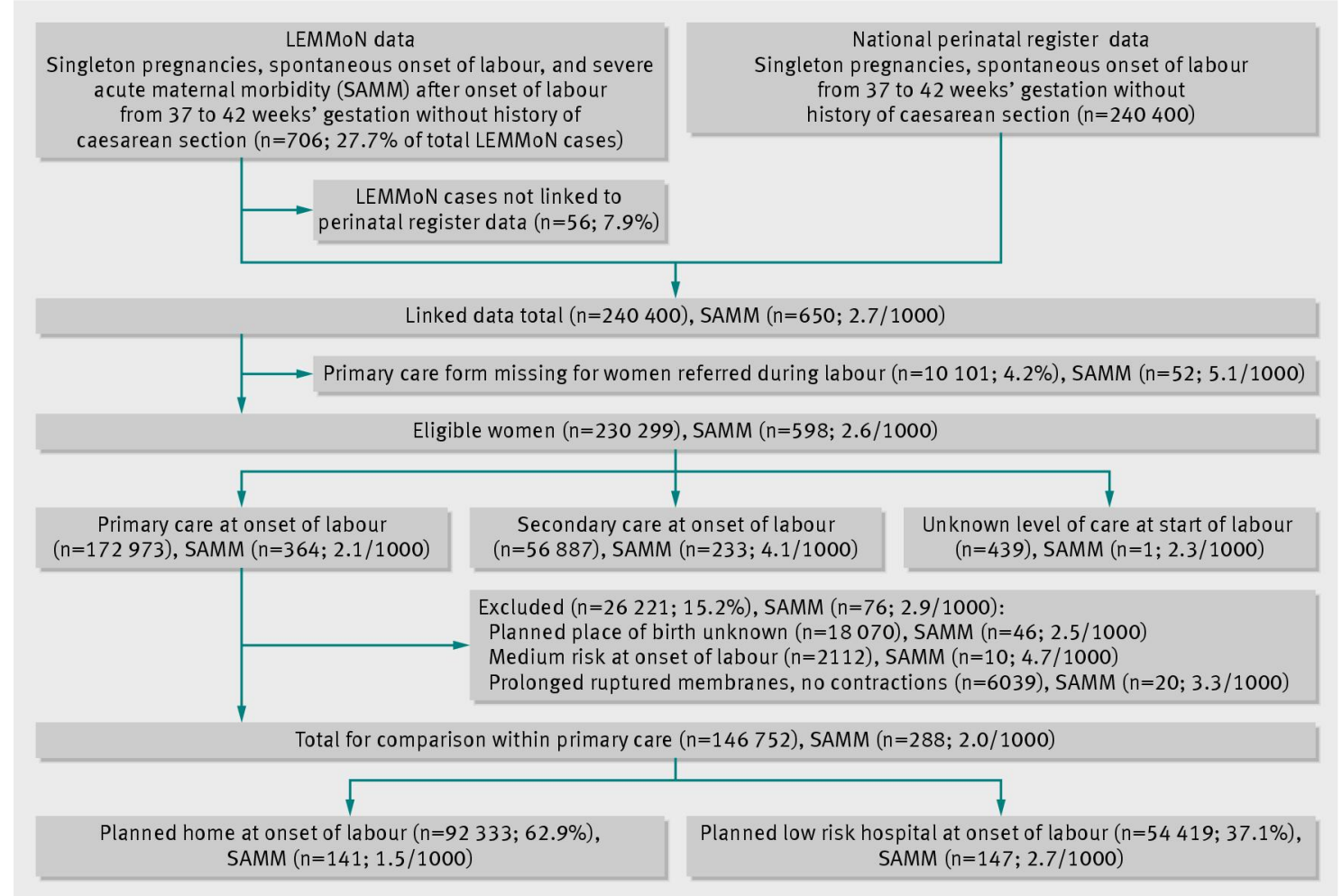

Flow of births between August 2004 and July 2006 